\section{Benign lichenoid keratosis: an off-center fold case}

\author{
Ashley Hamstra, Michael Messina, \\ Abel Torres \\ Department of Dermatology, Loma Linda \\ University Medical Center, CA, USA
}

\begin{abstract}
This off-center fold case depicts the difficult differential diagnosis for benign lichenoid keratosis. It is challenging to diagnosis this benign lesion through clinical exam, dermoscopy, and even dermatopathology. Given its similar appearance to regressed melanoma, it is important to be cognizant of both and up to date on the dermatopathology clues.
\end{abstract}

\section{Case Report}

A 53-year-old Caucasian female presented with an asymptomatic, changing pigmented lesion on her right forearm, first noticed two years earlier. Three months ago, one half had grown rapidly to $3.8 \mathrm{~cm}$ in diameter. It had initially become raised and scaly, before transitioning to flat and violaceous. The other half was brown and unchanged with central pink papules. She had not experienced pruritis, bleeding or burning throughout this progression. Her past medical history was significant for a meningioma in 1998. An enlarged right cervical lymph node was noted on physical exam at time of presentation to our office. The diagnosis was benign lichenoid keratosis.

\section{Microscopic findings and clinical course}

An excisional biopsy showed focal parakeratosis, epidermal thinning, dyskeratosis without atypia, irregular acanthosis, epidermal necrotic keratinocytes, vacuolar alterations of the basal layer, solar elastosis of the dermis, increased numbers of dilated blood vessels, a dense lichenoid infiltrate of lymphocytes at the dermoepidermal junction, numerous melanophages, pigment incontinence and a solar lentigo adjacent to the lesion. The S-100, Melan-A and HMB-45 immunohistiochemical stains were negative for melanocytes. The Ki17 stain showed equivocal active cells in the basal layer. This was suggestive of benign lichenoid keratosis vs. regressed melanoma. Further pathological review was sought from three dermatopathologists, who all interpreted the slides as benign lichenoid keratosis (Figures 1,2,3).

\section{Discussion}

Benign lichenoid keratosis (BLK), also known as lichen planus-like keratosis, is a common skin entity that typically presents as a solitary asymptomatic lesion on the trunk or distal upper extremities. There is an increased prevalence among women and Caucasians. ${ }^{1}$ It may be rough or scaly in texture and often transitions from pink to violaceous to hyperpigmented as its regression progresses. Multiple regression phases may be simultaneously present within a lesion. ${ }^{2,3}$

It is thought that BLK's may arise from a regressing seborrhoeic keratosis or a regressing solar lentigines. ${ }^{1,4}-6$ This particular case had remnants of a lentigo. While T-lymphocytes and cell-mediated immunity are predominant throughout regression, as inflammation advances the ratio of CD3 to CD20 diminishes. This indicates a relative increase in the percentage of B-lymphocytes and in humoral immunity. Autoantibodies may be produced by the multiplying B-lymphocytes. ${ }^{2}$

Clinically, a BLK resembles basal cell carcinoma, Bowen's disease, lentigo, seborrheic keratosis, actinic keratosis and, most importantly, melanoma, ${ }^{6}$ In addition to their clinical similarity, melanoma in situ may be dermoscopically indistinguishable from BLK in all stages of regression, especially if on the face, due to the face's unique epidermal structure. ${ }^{3}$ Furthermore if the regression is advanced, the remaining lesion may be insufficient to make a definitive diagnosis using dermoscopy, regardless of the site, due to pathological and procedural disruption of the lesion and distortion of much needed diagnostic attributes. ${ }^{6,7,8}$ The blue-white structures that are strongly associated with melanoma may be present in many other regressing lesions. ${ }^{4,9}$ Histological-ly, BLK's typically demonstrate epidermal acanthosis, parakeratosis and a band-like lichenoid lymphocyte infiltrate. Additionally there are clinical subtypes (classic, bullous, atypical, early and late) that further contribute to confusion and misdiagnosis. ${ }^{1}$

Distinguishing BLK from melanoma remains difficult. Many have noted certain findings that raise the suspicion of melanoma, such as proliferations of junctional melanocyte nests and Starburst giant cells. Also FISH may reveal altered melanocytes with an absent DNA copy number of chromosome $9 \mathrm{p} 21$. If any of these findings are present, taking a deeper section to rule out regressed melanoma is advised. ${ }^{8}$
Correspondence: Abel Torres, Loma Linda University Medical Center, Department of Dermatology, 11370 Anderson Street, Suite 2600, Loma Linda, CA 92354, USA.

Tel. +1.909.558.2890 - Fax: +1.909.558.2891.

E-mail: abelt@aol.com

Key words: benign lichenoid keratosis, melanoma, dermoscopy.

Received for publication: 10 August 2011. Accepted for publication: 11 August 2011.

This work is licensed under a Creative Commons Attribution NonCommercial 3.0 License (CC BYNC 3.0).

(C) Copyright A. Hamstra et al., 2011

Licensee PAGEPress, Italy

Dermatology Reports 2011; 3:e25

doi:10.4081/dr.2011.e25

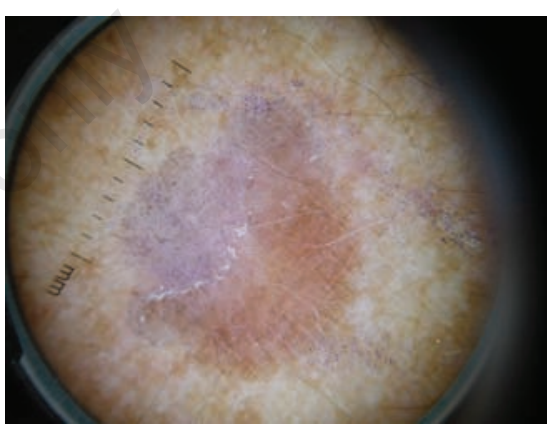

Figure 1. Dermoscopy.

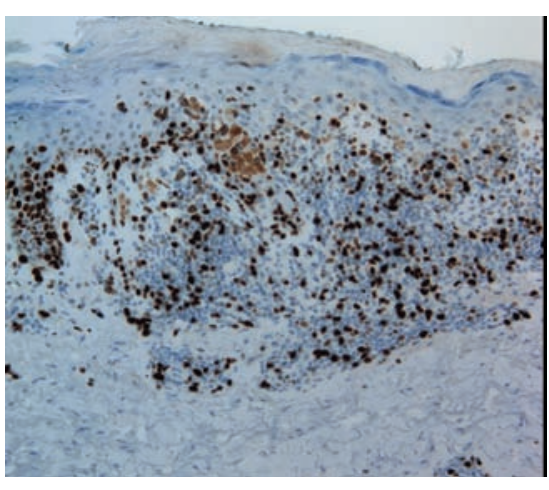

Figure 2. Ki67 Stain.

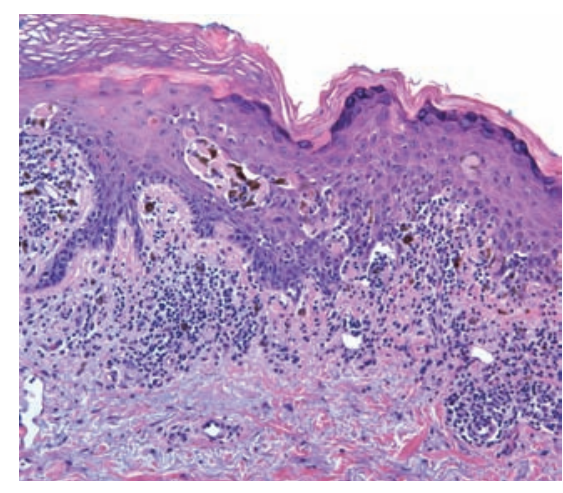

Figure 3. 20x slide. 


\section{References}

1. Morgan M, Stevens G, Switlyk S. Benign lichenoid keratosis: a clinical and pathologic reappraisal of 1040 cases. Am J Dermatopathol 2005;27:387-92.

2. Bayer-Garner I, Ivan D, Schwartz M, Tschen J. The immunopathology of regression in benign lichenoid keratosis, keratoacanthoma and halo nevus. Clin Med Res 2004;2:89-97.

3. Raptoulis G, Spencer R, Einstein B, et al. Lichen planus-like keratosis of the face: a simulator of melanoma in situ. Dermatol Surg 2007;33:854-6.

4. Zaballos P, Rodero J, Pastor L, et al. Dermoscopy of lichenoid regressing solar lentigines. archiv dermatol 2008;144: 284.

5. Zaballos P, Marti E, Cuéllar F, et al. Dermoscopy of lichenoid regressing seborrheic keratosis. Archiv Dermatol 2006;142: 410.

6. Bugatti L, Filosa G. Dermoscopy of lichen planus-like keratosis: a model of inflammatory regression. J Eur Acad Dermatol Venereol 2007;21:1392-7.
7. Giorgi V, Massi D, Salvini C, et al. Features of regression in dermoscopic diagnosis: a confounding fact? Two clinical, dermoscopic-pathologic case studies. Dermatol Surg 2006;32:282-6.

8. Dalton S, Fillman E, Altman C, et al. Atypical junctional melanocytic proliferations in benign lichenoid keratosis. Hum Pathol 2003;34:706-9.

9. Zalaudek I, Argenziano G, Ferrara G, et al. Clinically equivocal melanocytic skin lesions with features of regression: a dermoscopic-pathological study. Br J Dermatol 2004;150:64-71. 